\title{
VII.
}

\section{Beiträge zur Kenntniss der varicösen Hypertrophie der Nervenfasern.}

\author{
Von Dr. M. Roth, \\ Privatdocenten und Assistenten am pahologischen Institut zu Greifswald.
}

(Hierzu Taf. XII. Fig. 6-12.)

Seit Zenker (1) zuerst gewisse Veränderungen, welche gegenwärtig als varicöse Hypertrophie der Nervenfasern aufgefasst werden, in der Netzhaut des Auges beobachtet und H. Müller $(7,8)$ dieselbe richtig gedeutet, hat sich allmäblich eine grössere Zahl von Beobachtungen angesammelt, welche sich grösstentheils auf die Retina, zum kleineren Theil auf andere Abschnitte des Nervensystems beziehen. Die fragliche Veränderung besteht bekanntlich in einer spindel- oder keulenförmigen, seltener cylindrischen Verdickung des Axencylinders, womit sich meist ein grösserer Glanz, grössere Derbheit und Resistenz gegen Zusatzflüssigkeiten verbindet. Die Reactionsverhältnisse lassen darauf schliessen, dass an den verdickten Stellen der Eiweisskörper des Axencylinders noch vorhanden ist, aber in verschiedenen Stadien der Verdichtung sich befindet. Die Anschwellung tritt entweder in der Continuität der Faser auf und wiederholt sich nicht selten mehrmals, so dass die Faser ein perlschnurartiges Aussehen bekommt, oder sitzt der Faser endständig auf (letzteres ist indess meist als Kunstproduct anzusehen, da die Nervenfaser beim Zerzupfen lèicht abbricht). Die Varicositäten erscheinen homogen oder körnig; nicht selten, hauptsächlich in den grossen derben Formen, findet man einen oder mehrere kernähnliche Klumpen, wodurch manchmal eine grosse Aehnlichkeit mit Ganglienzellen bedingt wird.

Im Folgenden sind einige neue hierhergehörige Fälle mitgetheilt, die mir bei der Untersuchung einer grösseren Zabl von Rückenmarken und Augen vorgekommen sind. Es handelt sich dabei immer um zufällige Befunde, insofern als keine Erscheinungen intra vitam auf das Vorhandensein dieser Anomalie gedeutet 
hatten. Fall $\mathbb{I}$ bezieht sich auf die graue Substanz des Rückenmarkes, Fall II_VI auf die Netzhaut. Auṣ den angesteliten Experimenten geht hervor, dass die varicöse Hypertrophie sich auch künstlich an Thieren hervorrufen lässt.

Eall I. Verdickungen der Axencylinder und der verảstelten Fortsätze der Ganglienzellen in der grauen Substanz des Röckenmarks. Fig. 6-9.

E. A., 27 Jabre, Arbeitshäuslerin, Obd. (H. Prof. Grohe) 18. Februar 1870 ergab allgemeine Atrophie, ulceröse Bronchektasen der Lungen, frische Pleuritis sin., frische Diphtheritis des Ileum. - Zwischen Dura mater spinalis und Rückfläche der Arachn. zablreiche Adbåsionen. Aracho. wie bestäubt (Kleide Psammome), am unteren Theil einige Kalkplättchen. Rückenmark vou guter Consistenz.

Aus der Lendenanschwellung, welche einige Tage mit schwacher Chromkalilösung nach Deiters behandelt war, liessen sich die grossen Ganglienzellen mit Axencylinder und perästelten Fortsätzen schön isoliren. Die Zellen sind stark pigmentirt, auch an den Theilungsstellen der Fortsätze ist ofters Pigment abgelagert. An einigen verästelten Fortsätzen sitzen feinkörnige glänzende Anschwellungen (Fig. 9 a, b) die manchmal scharf unschrieben sind und einea hellen Fleck enthalten (a), so dass sie einem Kern mit Kerokörperchen ähnlich sehen. Oefter waren solche Verdickungen pigmentirt. Ferner fanden sich nehen normalen Axencylindern andre mit feinkörngen oder homogenen Anschwellungen, häufiger and stärlzer entwickelt in den Vorderbönern (Fig. 6) als in den Hinterhörnern (Fig, 7, 8). Uebrigens waren sie immer vereinzelt, nicht in Nestern wie man sie bäufig in der Netzbaut findet. Ihre Grösse war immer eine mässige (Maximum 0,05 Mm. lang; 0,01 dick bei $0,002 \mathrm{Mm}$. Dicke des zugehörigen Axencylinders). Dass es wirklich Axencylinder waren, ging daraus hervor, dass keide Theilungen an denselben vorkamen und ofter Reste der Markscheide ihnen anbafteten (bei: $c_{y} . c, d$ ).

Sonst worde nach Pigmentanhäufung in der Adventitia kleiner Gefässe bemerkt. Am gehärteten Präparat fand sich obliteration des Centralkanals durch rundliche Zellen, dagegen wurde jetat vergeblich auf die verdickten Nervenfasern gefahndet. Es kamen zwar Körper in der grauen Substanz vor, die Aehnlichkeit mit jenen Anschwellungen hatten dach komen es möglicherweise auch kleine Ganglienzellen oder Capillaren sein. - In der Peripherie der Hinterstränge zablreiche Corpora amylacea.

Diese Beobachtung vervollsiảndigt unsere Kenntnisse über die Verbreitung der varieösen Hypertrophie, die bisher nur aus der weissen Substanz des Rückenmarks bekant waren. Die Thatsache, dass an Axencylindern und an verästelten Fortsătzen analoge Verdickungen vorkommen, gibt eine neue Stütze für den von Max Schultze gefuhrten Nachweis, dass erhebliche Unterschiede zwischen den von Deiters aufgestellten Arten ron Ganglienzellenfortsätzen nicht existiren. 
Eine Beziebung zwischen dieser Anomalie und dern ibrigen Obductionsbefund ist vorläufig nicht zu erkennen.

Fall II. Hypertrophie der Nervenfasern der rechten Retina bei Verjauchung des Oberschenkels.

W. U., 36. Jahre, Arbeitsmann, hatte längere Zeit auf der medic. Klinik in einem typhösen Fieber gelegen. Obduction (H. Prof. Grobe) 5. März 1870. Grosser Decubitus auf dem Sacrum. Ausgedehnte Verjauchung der Weichtheile des rechten Oberschenkels und der Fossa iliaca mit frischer Blutung in die Jauchehöhle und Eröffnung des Hüftgelenks. Gelatinöse und käsige Infiltrate der Lungen. Milztumor. Leber und Nieren normal. Oedem der Pia mater cerebri.

Aus der mikroskopischen Untersuchung ist ausgedehnte Fettembolie der Lungencapillaren hervorzuheben.

Die Augen waren von der Art. ophthalm. injicirt und darauf in Müller'sche Flüssigkeit gelegt worden. Als nach zwei. Tagen die rechte Retina bei schwacher Vergrösserung durchgemustert wurde, fand sich etwas nach unten und innen von der Macula lutea ein kleiner in der Opticusschicht gelegener Heerd von Körnchenzellen und bypertrophischen Axencylindern. Beim Zerfasern kamen runde und spiadelförmige $(0,01 \mathrm{Mm}$. lang, 0,005 breit) verfettete Zellen zam Vorschein, manche davon waren kerubaltig. Ferner fanden sich länglìche glänzende Klumpen, die Aehnlichkeit mit Myelinformen hatten. Endlich sparsame varicöse Axencylinder von rundlicher Gestalt (bis 0,04 lang, 0,03 brei ) mit einem oder melreren kernähnlichen Körpern, von der Beschaffenheit wie sie bei Morbus Bright. und Leukämie bescbrieben sind. - Die Blutgefässe dieser Gegend, zum Theil blau injicirt, normal; von Fettembolie, wie ich sie in der Lunge gefunden, war hier nichts nachweisbar.

Retina im Uebrigen normal.

In der linken Retina zwischen Mac. lut. und Papille einige Corpora amylacea. Choroides beiderseits normal.

Fall III. Hypertrophie der Axencylinder in beiden Retinae bei multipeln Sarkomknoten der Unterleibsdrüsen. Hämorrhagische Peritonitis, Icterus catarrh.

J. R., 57 Jahre, Arbeiter, (Krankengeschichte nach gütiger Mittbeilung von Prof. Mosler) recip. den 14. April 1871, war vor zehn Wochen von heftigem Husten und Oedem der untern Extremitäten befallen worden. Vor 14 Tagen hatte sich gelbliche Hautfarbe hinzugesellt.

Stat us praes. Aeusserste Abmagerung, Icterus, Decubitus anf dem Kreazbein und dem linken Trochanter major, 0edem der unteren Extremitäten.

Physic. Untersuchung: am Thorax hinten links Ieichte Dämpfung und verschärftes Athmen; das nach unten an Intensităt abnimmt. Im Abdomen Ansammlung von Flüssigkeit, sehr bedentender Milztumor. Leberdämpfung reicht fast bis zum Nabel. - Temp. 39,4, Resp. 20, Puls 96, zwei grauweisse Stühle; Urin gibt mit $\mathrm{NO}_{5}$ deutliche Gallenfarbstoffreaction.

15. April T. 37,4 .

16. April T. 36,2 Collaps.

17. April T. 36,5, Collaps. nimmt zu, Trachealrasseln, fcterus hat zugenommen, Sopor. Tod Morgens 10 Ubr. 
Obduct. 18. April 1871. Abmagerung, leichtes Oedem der untern Extrem. und des Scrotum, Icterus der Hant und der innern Theile. Im linken Pleurasack c. 12 Unzen leicht getrübter gelbrother Flüssigkeit. Doppelseitige chronische Bronchitis mit cylindrischer Erweiterung der Bronchien. In der Bauchböhle 2 Quart trübe brauntothe Flässigleit, Grosses Netz atrophisch, mit sparsamen weissen derben Knötchen besctzt. Im Douglas'schen Baum linsengrosse weissliche und rötbliche Excrescenzen, stellenweise grốssere Plaques bildend.

Milz sehr gross, $10 \frac{1}{4}$ Zoll lang, $6 \frac{1}{2}$ breit, 4 dick. Oberfläche glatt, dunkelrotbbrawn mit zahlreichen linsen- bis thalergrossen rundlichen und zackigen Einlagerangen von dellgelber, zuweilen grauröthlicher Farbe. Auf dem Durcuschnitt zablreiche ähnliche Iinoten his $2 \frac{1}{2}$ Zoll Durchmesser von homogener oder undentlich faseriser Structur; Consistenz therall derb.

Nieren mit vereinzelten miliaren Kinötchen unter der Kapsel.

Retroperitonealdrüsen etwas vergrössert, derb, mit gelblichen circumscripten Einsprengungen, ebenso die Drüsen an der Porta bepatis.

Setieimhaut des Mlagens und Duodenum verdickt und geröthet.

Leber nicht vergrössert, mässig fesł. Durchschnitt dunkelbraun mif zahlreichen miliaren bis hanflorogrossen Fnötcben, welche icterisch sind, im Uebrigen mit den Knoten der Milz und Lymphdrüsen übereinstimmen.

Schleimhat des Dünn- und Dickdarms, Mesenterialdrüsen bieten nichts Besonderes.

Das Mark des rechten $0 s$ femoris von dunkeiblaurotker hämorrhagischer Beschaffenheit.

Die Knoten der Milz a. s. w. sind nach dern Urtheil des H. Prof. Grohe als Sarkome anzmsprechen. Der Hauptsache nach bestehen sie aus bündelweise verlaufenden, kurzen, nicht selten verfetteten Spindelzellen, dazwischen kommen auch, besonders in den Leberknoten, Haufen kleiner ruoder Zellen zam Vorscbein.

Linkes Auge: Glaskörper klar, leicht gelblich, consisteṇt; Choroides dünn, byperämisch. - Die grossen. Gefässe der Retina starli gefïlth, Gewebe im Ganzen von normaler Transparenz, nur zunächst um die Papille milchig trübe. Etwa gleich weit von Papille und Macula lutea, etwas nach oben von beiden, ein kleiner weisser Fleck, zwischen der nach aussen und oben verlaufenden Arterie und Vene gejegen. Bei schwacher Vergrösserung zeigt sich, dass der Heerd der Opticusschicht angebört, und zwar liegen nach oben (innen) einige spindel- and keulenförmige Körnchenzellen, mehr in der Tiefe (aussen) etwa dreissig mattglänzende homogene oder feingranulirte Körper, von denen die Mehrzahl einen kernäbnlichen glänzenden Klumpen entkält. In Heerd selbst und in der Nabbarschaft einige Corpora amylacea. Dicht daneben noch ein kleinerer Heerd, der dieselbe Anordnung und dieselben Elemente erkennen hasst. Beim Zerzupfen in Jodserum wurden meist kolbige und wenige cylindrisch verdickte Nervenfasern isolirt neben feinereren Varicositäten, die in ibrer Grösse und Beschaffenbeit nur wenig oder gar nipht von cadaverösen Varicositäten sich unterschieden. Blutgefässe und Böroerschichten normal.

Rechtes Auge. Glaskörper and Choroides wie links. - Im hintern obern ITmfang der Linse ein weisslicher Fleck. - Retina mit trübem Hof, $2 \mathrm{Mm}$. nach saten innen von der Macula latea und ebensoweit bach aussen von der Papille 
ein etwas grösserer weisser Fleck als links. Zerzupfen des in 0smiumsäure gehärteten Objects ergab denselben Befund wie links. Nur markirten sich hier noch eine Anzabl feiner Fetttröpfchen in Ganglienzellen dieser Stelle.

Fall IV. Hypertrophische Axencylinder bei doppelseitiger disseminirter Retinitis. Glaskörpertrübung. Delirium tremens, Bronchitis und Bronchopneumonie. Schwellang und Blutungen der Nieren.

C. H., 57 Jahre, Arbeitsmann, recip. 27. April 1871 (Krankengeschichte von H. Prof. Mosler). Klinische Diagnose: Pneumonia sin. crouposa, Delirium tremens. Patient früher immer gesund, bekam vor vierzehn Tagen nach Erkältung Schüttelfrost mit folgender Hitze, bald darauf Husten mit blutigen Streifen im Auswurf. Stat. praes. Temp. 40,2, Besp. 44, Puls 112, Bespirirt grösstentheils mit den Bauchmuskeln; links vorn Dämpfung, schwach bronchiales Athmen, vereinzelte Rasselgeräusche. Hinten bis zum Angul. scapul. Därppiung, bronchiales Atbmen, nach abwärts verschwächtes Athmen. - Herztöne schwach, Milz nicht vergrössert. Zunge trocken, viel Durst. Kein Husten und Auswurf. Kein 0edem. Urin röthlich, wolkig getrübt, mit $\mathrm{NO}_{5}$ leichter Albumengehalt. Auffallend ist der Tremor artuum. Nach Angabe der Stubengenossen redet Patient häufig irre. Ord,: Inf. Ipec. Chinín. Branntwein. Abends Delirien, Patient will das Bett verlassen. Nach Opium leidlich gate Nacht.

28. April T. 40, - R. 40, P. 116. Pat, aufgeregt, delirirt fortwäbrend, Wangen und Stirn heiss, Flockenlesen, Zittern der Hände, typhöser Zustand. Nervencentren durch Alkoholismus und Fieber erregt. - Heratöne voll, Impuls nicht deutlich wahrnehmbar. Links hinten leichıe Dämpfung, links vorn unbestimmtes Athmen. Rechts in der Regio supraclav, unbestimmtes, unterbalb vesiculäres Athmen. Urin concentrirt, spec. Gew. erhöht, geringe Spur Albumen, woraus auf acute Fluxion nach der Niere $z \mathbf{u}$ schliessen ist. Kein Gallenfarbstoff.

Patient empfindlich beim Berühren.

Prognosis infausta. Ord.: kalte Brause. Chinin.

Abends T. 39,8, R. 44, P. wegen Unruhe des Patienten nicht zählbar.

Patient delirirt fortwährend und ist nur durch hartes Anreden zum Antworten zu bringen. Worte sind nicht zu versteben. Physikalisch, Status idem. Herztöne nicht wahrnehmbar. - Chin. mit 0pium.

29. April: T. 39,6. R. 44. P. - Nacht schlaflos, Delirien, Zunahme des Tremor artuum. Subjective Symptome in fortwäbrender Steigerung, Flockenlesen, Stirn und Füsse kühl, mit Schweiss bedeckt. Bewusstsein nicht gänzlich geschwunden. Keine Lähmungserscheinungen. Untersuchung der Brust unmöglich. Kein Sputum. - Albumen des Harns um etwas vermehrt, ohne so reichlich zu sein, um auf tiefgreifende Störung der Nieren schliessen zu lassen. - Ord.: Warmes Bad. Kaffee. Arnica.

Trachealrasseln. Tod 4 Uhr Nachmittags.

Obduction 1. Mai 1871. Doppelseitige chronische Bronchitis mit cylindrischen Bronchectasien. Bronchopneumonische Stellen im rechten mittleren Lappen, alveolares Emphysem der Ränder, Hypostase und Oedem der binteren Abschnitte der Lungen. Schiefrige Induration der linken Lungenspitze. Braune Atrophie des 
Herzens. - Milz müssig vergroossert, weich, Durchschnitt abwechselnd hell und dunkelgrauroth.

Nieren etwas vergrössert, 230 und $220 \mathrm{Grm}$, sehwer. Kapsel zart, leich trennbar, Oberfläche glatt mit zablreiclen punktförmigen Extravasaten. Auf dem Durchsehnitt Rinde normal hreit, grauroth, Glomeruli deutlich. In der Marksubstanz jederseits ein kleines Fibrom. Consistenz der Nieren etwas vermehrt.

Leber vergrössert, $2030 \mathrm{Grm}$. schwer, leichter Grad won interstitieller Hepatitis. - Chroniscber Magenkatarrb.

Die Augen konnten erst 3 Tage p. m. herausgenommea werden.

Links: Glagkörper noeh cohärent, etwas trübe; enthält zahlreiche blasse Zellen verschiedener Grösse mit 1-2 Kernen. Die Zellen sind theils rund, theils mit kurzen Fortsätzen versehen, manche enthalten Vacuolen.

Linse gellbich, istar.

Retina diffus getrübt, am intensivsten im oberen äusseren Viertel bis gegen den Aequator. Die grossen Gefässe mässig gefüllt, spitzen sich gegen die Papille zir. In der getrübten Partie bis $10 \mathrm{Mm}$. Entfernung yọ der Papille eine grössere Zahl weisser Punhte, sie liegen vorwiegend nach oben und aussen, wenige nach anten von der Macula lut, dazmischen einige Extravasate. Längs eines nach unten aussen verlaufenden Gefässes ein weisser Streif.

Epithel der Choroides leicht ablösbar, rostbraun. - Choroides dünn, blass. braun, im hinteren ausseren Umfang violett. Mikroskopisch an letzterer Stelle Hyperämie ohine weitere Veränderung.

Rechts: Glaskörper etwas trübe; mikroskopisch wie links.

Linse gelblich, vorn in del Mitte ein weisser Fleck.

Retina rings um Macula lut. trübe. Gefässe spitzen sich gegen die Papille zu, an zwei nach aussen verlaufenden Gefässen ein weisser Streif bemerkbar. In der trühen Partie der Retina and etwas darüber hinaus bis $8 \mathrm{Mm}$. von der Papille vereinzelte weisse Flecke, nach oben yon der Papille ein streifiger Bluterguss.

Choroid. dưna, hellbraun, nach aussen und nach innen je eine grössere violette Stelle.

Mikroskopisch fanden sich in dem grössten Fleck unterhalb der Mac. lut. links zahlreiche meist langspindelförmige Varicositäten, ohne kernähnliche Abscheidung, sowie einige Rörnchenzellen und fettige Entartung der Capillargefässe. Derselbe Befuod in mehreren kleineres Heerden in der Nähe der Macula, während die peripherischen Flecke blos körnchenzellen und verfettete Capillargefässe enthalten. Die grossen Gefässe zeigen ziemlich stark verdickte Wandungen.

Aehnlich war der Befund im rechten Auge.

Die Nieren, am erbärteten Präparat untersucbt, zeigen normal entwickeltes Stroma, Glamerali ind umspinanende Capillargefässe hyperämisch. Die punktförmigen Extravasate der Oberflache entsprechen Aohäufungen von rothen Blutkörperchen im Lumen gewundener Harnkanälchen. Dio Epithelien der letateren feinkörnig getrübt, die Zellgrenzen meist undeutlich, anch die Kerne oft verdeckt; vereinzelte hyaliae Cglinder. In den Papillen zahlreiche derbe Cylinder.

V. Fypertrophische Azencylinder der linken Retina in der Umgebung eines 
frischen Blotergusses. Circumscripte eitrige Choroiditis; metastatische Heerde der Lungen und Nieren bei jauchiger Koiegelenksentzündung.

F. D., 24 Jahre alt, Schäfer, auf der medic. Rlinik einige Monate an doppelseitiger Inniegelenksentzündung behandelt, starb unter pyämischen Erscheinungen.

Obduction am 8. November 1871 (H. Prof. Grobe). Decubitus auf dem Sacrum. Verjauchung beider Kniegelenke, rechts (Punction intra vitam) mit Perforation in die Weichtheile des Oberschenkels, frisches Blutcoagulum im Recessus. Links ebenfalls Perforation des Gelenks nach dem Oberschenkel und ausgedehnte Senkung nach der Wade. Circumscripte eitrige Perichondritis des 7. rechten Rippenknorpels. Lobuläre Infiltrate und Abscesse der Lungen mit doppelseitiger fibrinöser Pleuritis. Miliare Heerde der Nieren. Geringe Schwellung der Milz.

In den eitrig infiltrirten Stellen der Lungen und Nieren fand Fr. Prof. Grohe die neulich von Klebs, von Recklinghausen und Waldeyer beschriebenen Nester von Vibrionen (Monaden) in exquisiter Entwickelung.

Die Untersuchung der Augen ergab:

Rechts halbmondförmige Trübung am inneren Rande der Papilla n. opt., sonst nichts Abnormes.

Links gelbliches 0edem an der unteren Uebergangsfalte der Conjunctiva. Cornea, Iris, Linse normal.

Glaskörper klar, baftet im hinteren Umfang der Retina ziemlich fest an. Retina nach aussen von der Mac. lut., nach innen von der Papille etwas trübe. Grosse Gefâsse schwach gefülit, nur zwei nach innen verlanfende Stämmchen erweitert und hyperämisch. Zwischen beiden, $5 \mathrm{Mm}$. nach innen von der Papille, ein stecknadelkopfgrosser frischrother Bluterguss, ringsherum ein schmaler getrübter Hof, nach aussen und innen schimmert etwas Bläuliches aus der Tiefe durch. Beim Abheben der Retina liegt zwischen ihr and der Choroides ein gallertiges mit rothen Streifen durchsetztes Gerinnsel, das sich von der Blutungsstelle gegen die Papille erstreckt.

Pigmentepithel hellbraun, wohlerhalten, fehlt nur über ejnem hirsekorngrossen weisslichen Knötchen der Choroỉes. Letzteres entspricht in seiner Lage genau dem Extravasat in der Retina. Tm dasselbe findet sich eine dunkelrothe Zone in der sonst bellbraunen mässig bluthaltigen Choroides. Bei Loupenvergrösserung erscheint das Pigmentepithel am Rande des Knötchens von dunkler Farbe, rings um dasselbe sind die grösseren Blutgefässe dilatirt, geschlängelt und byperämisch. Das Knötchen besteht, wie Durchschnitte am gehärteten Präparat ergaben, aus dichtgedrängten leicht verfetteten Eiterkörperchen, deren Zahl am grössten in der Capillarschicht, nach aussen etwas geringer ist. Gefässe und Pigmentzellen fehlen in diesem Heerde fast völlig, Anhäufungen von Vibrionen wurden nicht gefunden.

Das subretinale Gerinnsel enthielt in einer glasigen zähen Grundmasse zablreiche rothe Blutkörperchen, vereinzelte ein- und zweigliedrige Körperchen (Monaden?), Pigmentepithelien und gequollene Stäbchen und Körner der Retina.

Ein Stück der Netzhaut mit der Blutung wurde in Osmiumsäure gebärtet und in eine Anzahl Schnitte zerlegt. Die rothen Blutkörperchen finden sich in grösserer Masse zwischen der Opticusschicht und der M. limit. interna, welche bauchig gegen den Glaskörper vorgewölbt erscheint. Die Opticusfasern.meist wohl- 
erhalten, nur auf einem Präparat waren sie zerrissen, die Enden in die äusseren Schichten urngeschlagen and einige davon mit homogenen Varicositäten besetzt. Die äusseren Schionten sind pon Blutkörperchen durchsetzt und zerrissen, die Stäbchenschicht fehlt. (Der Bluterguss zwischen Retina und Choroides stammte also ans der ersteren.)

In der Nähe der Blutung, und zwar gegen die Papille bin, vereinzelte oder in kleinen Groppen stehende Varicositäten in der Optiousschicht. Die Anschwellungen sind alle von mässiger Grösse, durch Osmium hellbraun gefärbt, obne Abscheidung im Innern. Capillargefässe dieser Gegend meist leer, die Wandung grösserer Gefässe etwas verdickt. Keine Spur von Verfettung.

Im Glaskörper vereinzelte rothe Blutkörperchen.

V1. Hypertrophische Asencylinder der linken Retina. Decubitus. Yerjauchung des linken Oberschenkels. Putride Bronchitis.

C. R., 48 Jahre alt, Arbeiter.

Obdaction 11. December 1871. Extreme hmagerung. Decubitus. Ausgedehnte Verjauchung des tiefen Zellgewebes am linken Oberschenkel bis gegen das Sacrum, ohne Betheiligung des Knochens, mit mehreren spontanen Perforationen and Incisionswunden. Oedem der rechten unteren Extremität mit verbreiteter Thrombose der Oberschenkelvenen. In beiden Lungen Bronchectasien mit stinkendem Inhalt (mikrosk، Fettnadeln, zerfallende Eiterkörperchen, Monaden). Frische lohuläre Pneumonie der rechten Lunge mit beginnendem putridem Zerfall. - Milz klein, schlaff. - Nieren in der Rindensubstanz grauröthlich, etwas trübe. Leber: rechter Lappen mit Zwerchfell verwachsen, Durchschaitt dunkelbraun, Zeichnung der Acini verwaschen.

Die Bulbi, an denen zunächst nichts Auffallendes bemerkt worden, warden einige Tage in Müller'sche Flüssigkeit gelegt. Bei nochmaliger Betrachtung markirte sich in der linken Retina etwas nach oben und aussen von der Papille ein weisslicher Fleck. Hier fanden sich bei schwacher Vergrösserung oberflächlich (nacb innen) liegende rundliche und lăagliche kö́nchenzellen im Winkel zwischen zwei grösseren Gefüssen. Beim Zerfasern wurde noch eine grosse Zahl von hypertrophischen Nervenfasern sichtbar (bis $0,06 \mathrm{Mm}$. Länge, 0,03 Breite), obne oder mit (1 oder 2) kernähnlicben Abscheidungen. Auch roseakranzörmige Elemente, wo an einer Faser alle Uebergänge von kleinen zarten bis zu zrossen derben kernhaltigen Varicositäten vorkamen, waren vorbanden. Einge rundiliche Körper von derselben Beschaffenheit wie die grösseren Varicositäten, waren wohl durch Abbrechen der zugehörigen Nervenfasern frei geworden. In den Körnclienzellen war nicht selten ein Kern sichtbar. Eituge Capillargefässe mit sparsamen Fettröpfchen besetzt. Im Heerd selbst und an einiger Entfernugg davon mebrere grosse mehrfach geschichtete Corpora amylacea (bis 0,036 Durchm.).

An der Choroides aichts Aufallendes.

In rechten Auge bein analoger Heerả. Auf senkrechten Schnitten in der Nähe der Macula lutea erschienen die inneren an die Zwischenkörnerschicht grenzenden Elemente der äusserep Körnerlage etwa um das Doppelte vergrössert, homogen und stark glänatend. 
In den 5 letzten Fällen (ILVI) sind circumscripte Heerde, in geringerer oder grösserer Zahl (IV), ein- oder doppelseitig, in der Retina vorhanden, wie sie verbältuissmässig häufig bei Morbus Brightii, bei jntracraniellen Krankbeiten, seltener bei Leukämie und Syphilis beobachtet sind. Die Heerde bestehen jeweilen aus veränderten Nervenfasern, Körnchenzellen, verfetteten Capillargefässen, Corpora amylacea und Extravasaten in verschiedener Combination. In Fall IV fand sich gleichzeitig partielle Hyperämie der Choroides und Zellenneubildung im Glaskörper. Fall $V$ weicht insofern von den übrigen $a b$, als der retinale Heerd secundär über einer kleinen Eiteransammlung in der Choroides entstanden ist.

In ätiologischer Beziehung bieten diese Beobachtungen manches Interessante.

Zunächst gehören Fall II, V und VI wegen der Verwandtschaft der zu Grunde liegenden Prozesse zusammen. In allen 3 finden wir nehmlich ausgedehnte brandige und jauchige Zustände, jauchige Zellgewebsentzündung einer (II, VI) oder beider Unterextremitäten ( $\mathrm{V}$, mit Ausgang von den Kniegelenken), wozu in allen Decubitus, in einem noch putride Bronchitis hinzukommt. Embolische Prozesse sind in Fall II, abgesehen von der Fettembolie der Lungen, nicht vorhanden. Auch in Fall VI fehlen embolische Heerde. Fall V dagegen zeigt gemischten, septisch-embolischen Charakter: neben jauchiger Beschaffenheit der Krankheitsheerde in den unteren Extremitäten fanden sich muJtiple metastatische Eiterungen, von denen die in den Lungen und Nieren, wie Herr Prof. Grohe nachwies, körniges, wahrscheinlich importirtes Material (Monaden) beherbergten, während es für den Eiterheerd der Choroides, welcher die Retinalblutung zur Folge hatte, nicht gelang, körniges Material in grösseren Quantitäten aufzufinden.

Da nun bisher analoge Heerderkrankungen der Retina fast nur (über die traumatische Entstehung s. unten) als Folge anderweitiger Krankheiten beobachtet sind, so wird man auch diesmal die Retinitis mit dem Allgemeinprozess in nähere Verbindung setzen dürfen, und könnte von ätiologischem Standpunkt wie eine Ret. brightica, leukaemica etc. auch eine Retinitis septica statuiren.

Wie bei anderweitig entstandener secundärer Netzhautaffection scheint auch bei septischer Grundlage dep Prozess sich zuweilen auf Extravasatbildung zu beschränken. Dahin gehört folgender Fall: 
YII. Retinalblutungen bei Bronchitis putrida duplex etc.

C. T., 24 Jahre alt, Iutscher. Obduction 18. April 1870. Doppelseitige putride Bronchitis. Linke Lunge grossentheils adhärent, an der Basis eine mehr als faustgrosse Jauchehöhle. Auf dem Durchschnitt zahlreiche bronchopneumonische zum Theil in jauchigem Zerfall begriffene Infiltrate, - Ecchymosirung and frischer Faserstoffbeschlag der rechten Pleura. - Pericardialfițssigkeit getrübt, Pericard an der Herzbasis aufelockert nod ecchymotisch.

Mässiger Milztumor.

Parenchymatöse Scluwellung der Nieren und Leber.

Mikrogkopisch fanden sich in den Bronchien and Höblen der Lungen fettige Eiterkörperchen, Pigmentliörner, Fetlnadeln, masșenhafte Monaden.

In der Retiva des rechten Auges ein frischrothes streifiges Extravasat nach innen und unten von der Macula lutea, Mikroslropisch wohlerhaltene rothe Blatkörperchen, die sämmtlich in der Opticusschicht liegen. An der Blutungsstelle und in der weiteren Umgehang der Macula lutea leichte Verfettung einiger Capillaren, Sonst nichts Abnormes.

Link drei Apoplexien, eine länglich parallel dem Faserverlauf, $1 \mathrm{Mm}$. nach unten und innen von der Papilla $\mathrm{n}$, opt., die zwei anderen rundlich, 0,5 Mm. gross, auf dem inneren und äusseren Umfang der Macula lutea. - Die streifige Blutung sitzt in der Opticussebicht, die ingeren Enden der Müller'schen Fasern glänzen bier etwas mehr als gewöhnlich. Die runden Extravasate gehöreo der Ganglienschicht an, die darüher weglaufende dünne Opticusschicht ise frei von Bluthörperchen.

Keine anderweitigen Veränderungen.

In. der Literatur ist mir ein Hinweis auf Veränderungen der Retina bei septischen Prozessen nicht aufyestossen; wenn nicht vielleicht die Notiz von Virchow (Archiv X. S. 173) hierhergehört, dass sich bei einer an Gangraena senilis gestorbenen Person rothe Flecke in der Relina fanden.

Ueber den Zusammenhang der Netzhautveränderung mit dem Hauptprozess im Fall III und IV sind, bis weitere Beobachtungen zur Aufklärung beitragen, nur Vermuthungen möglich. ln Fall III könnte man an eine Infection des Blutes von den Sarkomen aus denken, welche möglicherweise zur Netzhautaffection gefuhrt hat. In Fall IV wäre, bei der relativ geringen Alteration der Nieren, vielleicht der acute Alkoholismus anzuschuldigen.

Meistens ist die Hypertrophie der Nervenfasern mit anderweitigen Veränderungen verknüpft. Unter 35 die Retina betreffenden Fälen $(1-30$, II-VI) finden sich, abgesehen von zwei aphoristisch gehaltenen Mittheilungen $(19,20)$, jedesmal Extravasate, verdickte oder verfettete Blutgefässe, Körnchenzellen, Eiterkörperchen, Hypentrophie der Radialfasern etc. in verschiedener 
Combination vor. In vielen Fällen tragen die Veränderungen in ihrer Totaliät einen deutlich irritativen Charakter. Nicht selten findet auch eine Retheiligung der Choroides und des Glaskörpers statt.

Aus dieser Constanz der Combination ist aber keineswegs der Schluss zu ziehen, dass die Hypertrophie der Nervenfasern immer secundär entstanden, eine Folge der Veränderungen des Bindegewebes und der Blutgefässe sei: denn für's Erste fehlen manchmal unter sonst ähnlichen Verhältnissen die Varicositäten gänzlich, wie Virchow, H. Müller und Schweigger mit Recht hervorgehoben, und wolür wir auch unter unsereu septischen Fällen ein Beispiel (VID) gefunden haben; sodann ist in einzelnen Fällen die varicöse Hypertrophie so überwiegend äber die Veränderung der Umgebung, dass man letztere blos als nebensächliche Complication betrachten kann, eine Anschauung, die schon Virchow (Archiv ${ }^{\dagger} X$. S. 177) ausgesprochen hat. - In dieser Richtung verdienen auch einige Fille aus dem Centralnervensystem unser Interesse. So waren nach Hadlich (35) die Varicositäten diffus in der Corticalsubstanz des kleinen Gehirns verbreitet und daneben fand sich als einzige Abnormität Verfettung der Gefässe. In dem Fall von Frommann (37) sind zwar hochgradige interstitielle Veränderungen im Lendentheil, dagegen in der P. dorsalis des Rückenmarks kleine Heerde von verdickten Axencylindern als wesentliche Anomalie angeführt (vgl. auch Beob. I). Allerdings finden sich aber sehr häufig gleichzeilig ausgedehnte Veränderungen der Neuroglia mit mehr oder weniger ausgesprachen entzündlichem Charakter, so dass auch hier die Hypertropbie der Axencylinder meist nur eine Theilerscheinung eines complicirten Prozesses ist.

Aus dieser Betrachtung folgt, dass die Aetiologie der varicösen Hypertrophie in der Netzhaut im Wesentlichen mit der der Retinitis zusammenfällt. Man kann demnach unterscheiden:

1) Retinitis (und varicöse Hypertrophie) als Theilerscheinung von Blutkrankheiten verschiedener Art; am hıäufigsten, meist doppelseitig und in multiplen Heerden. (Ret. brightica ete.)

2) Retinitis (und varicöse Hypertrophie) als Folge einer Krankheit des Gehirns oder der Gehirnhäute, indem ein entzündlicher Prozess discontinuirlich oder fortgeleitet auf der Bahn der Nervi optici in der Netzhaut auftritt. Ebenfalls bäufig, doppelseitig, meis ț in diffuser Form, selten in multiplen Heerden. (Neuritis opt.) 
3) Chronische Choro-Retinitis mit Ausgang in Atrophie und pigmentirung der Netzhaut (Retinitis pigmentosa). Dahin gehört ein Fall von Wedl (17) mit beerdweise verdickten Nervenfasern bei Atrophie der abrigen Optjeusschicht.

4) Primäre Retinitis. Dahin gehören zwei. Fälle, durch Trauma entstanden, wo bypertrophische Nervenfasern zur Beobachtung kamen. Berlin $(23,24)$ beabachtete in 2 wei wegen Verletzung enucleirten Augen an der Rịssstelle dér Retina bedeutend verdickte Nervenfasern ${ }^{2}$ ).

Der varicösen Hypertrophie kommt nach alledem keine specifische Bedeutung für einen bestimmten Prozess za, wie dies H. Müller (9) für den Morbus Brightii annahm, sie ist vielmehr eine mehr oder weniger hervorragende Theilerscheinung eines auf allgemeiner oder localer Ursache beruhenden Entzündungsprozesses.

Die Zeitdauer, welche zur Entwickelung der Hypertropbie nothwendig ist, lässt sich nur selten angeben, weil meist der Beginn des Prozesses nicht genau zu bestimmen ist. Einige Beobachtungen zeigen indess, dass dies in viel kürzerer Zeit geschieht, als man vielleicht a priori vermuthet hätte. In einem Fall (Berlin 24) waren sie schon 17 Stunden nach Verletzung der Petina an der Zerreissungsstelle vorhanden, wenn auch von mässiger Zabl und Grösse; in einem anderen (Berlin 23), 2 Tage nach der Verletzung,

1) Nach diesen Gesichtspunkten geordnet ergibi sich für die varicöse Hypertrophie der Retina aus 3ว Fällen folgendes Häuligheitsyerhältniss, das natürlich aur provisorische Gültigkteit hat:

1. Alterationen des Blutes . , . . 22

Morbus Br. $\quad 12(1.2 .3 .5 .6 .8 .9 .10 .11 .12 .16 .28)$

Syphilis . 1 (19)

Leuktaic . . $2(18.29)$

Septicämie , 3 (II. V. VI)

Pyamie . . 2 (4.22)

Alkoholismus . 1 (IV)

Milzsarkam elc. 1 (III)

II. Gehirnkrankheiten verschiedener Art 8 (14.35.20.21.25.26.27.30)

III. Retinitis pigment. . . . . . 1 (17)

IV. Metinitis idiopath. (tranmat.) . . $2(23.24)$

V. Angen unbekannten oder unsicheren

Ursprungs $\cdot . \cdot . \quad 2(7.13)$ 
fanden sich zahlreiche und grosse Varicositäten, so dass die Opticusschicht dadurch bedeutend verdiclit erschien, auch besassen sie grösseren Glanz, aber noch keine kernabhnlichen Abscheidungen. Berlin bat mit Recht auf diesen Befund Gewicht gelegt. Indess auch die kernähnlichen Körper können sich unter Umständen rasch entwickeln; so fand Virchow einmal (3) 11 Tage nach Beginn der Krankheit, wo doch wahrscheinlich der Prozess in den Augen erst einige Tage später begonnen hatte, bereits ausgebildete derbe kernhaltige Anschwellungen. In Fall IV dagegen, wo der Grundprozess 14-16 Tage alt, die Retinitis aber möglicherweise viel jünger war, fanden sich nur einfach angeschwollene Nervenfasern. - Bei chronischem Verlauf scheint es in den meisten Fällen zur Sklerosirung und Bildung von kernähnlichen Abscheidungen zu kommen. ob dies immer gesehiebt und wie lange die hypertrophischen Axencylinder bestehen können, ist nicht bekannt (vgl. unten Experimente); ebenso nicht ob eine Rückbildung zum "Normalzustand vorkommt.

Auch im Gehirn braucht es bis zur Ausbildung relativ grosser, aber weicher kernloser Varicositäten nur wenige Tage (Encephalitis neonator.); im Rückenmark fand W. Müller (40) grosse, aber homogene Anselswellungen der Axencylinder am 13. Tage nach einer Verletzung. Ganz grosse sklerotische formen wie in der Retina und insbesondere die kernähnlichen Abscheidungen sind sowohl im Gehirn als im Rückenmark bisher nicht beobachtet worden.

Welche functionellen Störungen die Verdickung der Nervenfasern mit sich führt, darüber liegen meines Wissens noch keine unzweideutigen Beobachtungen vor.

Experimentelles. Es lag nahe die Erfahrungen von Berlin über traumatische Entstebung der Varicositäten an Thieren durch Verletzung der Retina zu controliren. Es wurden dazu zwei Hunde und eine grössere Anzahl Kaninchen verwendet. Die dazu erforderliche Operation ist einfach und unblutig: man schneidet den Conjunctivalsack oben oder aussen ein, trennt den Ansatz des Musc. rectus sup, oder extern., ziebt sodann den Bulbus mit einer Pincette nach unten oder innen und sticht mit einer Nadel möglichst in der Nähe der Eintrittsstelle des Nerv. optic., wo die Nervenfasern am zahlreichsten sind, einige Linien tief ein. Es entsteht dadurch ein kleines Loch in der Retina, dessen Umgebung beliebige 
Zeit nach der Verletzung untersucht wird. Einen grösseren Substanzverlust erhält man durch Anwendung einer glühend gemachten Nadel, im Uebrigen ist das Resultat dasselbe. Yon der Methode, einen Faden durch den Bulbus zu ziehen und liegen zu lassen, was zuerst versucbt wurde, bin ich später abgekommen, weil die verletzte Stelle oft nicht ohne Zerreissung ron dem Faden gelöst werden kann und die excessive Eiterbildung in Glaskörper die mikroskopische Untersuchung beeintedichtigt.

Diese Nachtheile fallen bei Stichverletzangen weg: die Reaction ișt in allen Theilen sebr gering, am geringsten auffallenderweise in der Choroides, wo ausser circumscripter Enträrbung des Bindegewebes und der Epithelien blos vereinzelte körnchenzellen wahrgewormmen wurden. Der Glaskörper in der Nähe der Terletzung enthält Blutlörperchen, Theile der zerstörten Netzhaut und Choroides, ferner eine mässige Zaht neugebildeter farbloser, zuweilen pigmentirter Zellen, wathrend die "Intercellularsubstanz streifig oder körnig getrübt erscheint.

Die Retina bleibt in allen Fälen durehaus trapsparent, selbst an den Wundrầndern, welche gegen den Glaskörper umgeschlagen zu sein pflegen; abgesehen von etwa aufliegenden Blutcoagulis oder blutiger Infiltration der Ränder. Die Blutgefässe, welche beim Raninchen bekanntich auf die Markbuschel beschränkt sind, erseheinen in frischen Fällen erweitert und hyperämisch. MiLroskopisch findet man eine blasige Abhebung der Adventitia rom Gefässstambi (Oedem), spater eine zellige Verdickung der Gefässwand. - Die Untersuchung der Cmgegend der Stichöfnung ergibt immer Ablagerung dunklen Pigments (aus der Choroides) in den äusseren Schichten der Notzbaut; in der Nervenfaserschicht, wenn ein Gefäss verletat warde, Blutkörperchen ader gelbbraune Pigmevtklumpen; băufig raricöse Verdickungen an den durchrissenen Nervenfaseru ring tum den Wundrand; seltenes findet raan dergleichen in eiager Enferang von der Verletzung miten unter wohlerhaltenen Nerventhsera. Besonders instructiv sind die Bilder, wern markhaltige Nervenfasem verletzt wurden: man siebt datn ricbt selten den perdickten Axencylinder noch innerhalb der augequollenen Markscheide liegen oder kann die Anschwellung bis zum Uebergang in eine durkelcontonrirte Nervenfuser verfolgen (Fig, 12), Durch Carmin Hiss sich das Verhalter noch ansehalicher machen, 
da das Mark darin unverändert bleibt, während die Anschwellung blassroth gefärbt wird. Frisch in Jodserum sind die Anschwellangeu bald homogen, bald feinkörnig, obne Atudeutung einer streifigen Textur, von weicher Consistenz und nur mässigem Glanz; untersucht man sie dagegen, nachdem einige Tage Müll er'sche Flüssigkeit eingewirkt hat, so erscheinen sie glänzend, derb und zerbröckeln beim Zerzupfen leicht.

Solche Varicositäten finden sicb ein bis 10 Tage nach der Verletzung und scheint in dieser Zeit nur eine mässige Vergrösserung, zugleich eine gröbere Granulirung derselben aufzutreten. Weitere Entwickelungsformen wurden nicht beobachtet; ebenso nicht Verfettung. Da aber Varicositäten schon 16 Tage nach der Verletzung sehr sparsam waren und in noch späteren Terminen Nervenfasern um die stichöffrung überhaupt nicht mehr vorkommen, so muss eine vollständige Rückbildung dieser Formen stattinden, deren Zustandekommen zu beobachten mir nicht gelungen ist.

Schon in den ersten Tagen beginnen im Wundrande Veränderungen des interstitiellen Bindegewebes. Man bemerkt ein Aufquellen und stärkeren Glanz der inneren Enden der Radialfasern, zuweilen Vermehrung der Kerne der letzteren und vereinzelte Körnchenzellen. Die Hauptsache leistet das in der Opticusscbicht parallel den Nervenfasern verlaufende Bindegewebe. Schon nach 2 Tagen begegnet man am Wundrande einigen derben, spindelförmigen oder mannigfach verästelten Zellen, deren lange fadenförmige Ausläufer leicht mit Nervenfasern verwechselt werden können. Im Innern der Zellen liegen 1-2 grosse granulirte Kerne. Diese Zellen vermehren sich in der nächsten Zeit bedeutend, so dass schon nach 16 Tagen der Wundrand eine derbe narbige Beschaffenheil besitzt, während nervöse Elemente, wie erwähnt, in dieser Zeit bereits spärlich geworden sind. Auch fettige Degeneration mancher Bindegewebszellen findet sich in dieser. Zeit. -

Der Substanzverlust in der Retina ersetzt sich nie, die Wundränder glätten sich vielmehr aus, erscheinen, durch Atrophie der äusseren Schichten, zugeschärft und nach aussen (gegen die Choroides) umgebogen. Im Rande findet sich nur derbes faseriges Bindegewebe und Pigment ohne nervöse Elemente. Das Loch in den 3 Häuten des Auges wird verlegt durch einen glasartigen Pfropf, der aus hyaliner oder streifiger Grundsubstanz mit eingestreuten 
Zellen besteht und von der Sklera und dem Glaskörper gemeinschaftlich geliefert wird. Retina und Choroides sind nicht daran betheiligt. Das Choroidealepithel baftet dem zugeschärften Netzhautrand fest an, die Zellen sind von ungleicher, oft sehr bedeutender Grösse, platt, wenig oder gar nicht pigmentirt, zuweilen verfettet. Die Choroides selbst ist local entfärbt und besteht aus derbem faserigem Bindegewebe. Diesen Zustand fand sich bei einem Kaninchen 176 Tage nach der Verletzung.

Es muss nun bemerkt werden, dass nicht in allen Experimenten varicöse Nervenfasern gefunden wurden. Diès mag zum Theil auf einem Fehler in der Untersuchung beruben, da die Reaction, wie gesagt, ausserordentlich gering ist, und die kleinen Heerde varicöser Fasern leicht entgehen können. Ziebt man die möglicherweise oder gewiss unzureichend untersuchten Falle ab und diejenigen, wo schon complete Vernarbung eingetreten ist, so bleiben 9 unten mitgetheilte Experimente, von denen 6 eir positives Resultat ergeben haben.

Exp. X\%. 22. November 1871. Grosses graues Raninchen.

1) Stich in den linken Buhbus mit glühender Nadel. Tod nach 28 Stunden. Lijcke der Retina laphtkorngross, $4 \mathrm{Mm}$, von der lapille, fallt zum Theil in die marlihaltige Zone. - Resultat negativ.

2) Einfacher Nadelstich in den rechten Bulbus, Tod gach 28 Stunden. Hierza Flg. 12.

Die Stichwande liegt $1 \mathrm{Mm}$. von der Papille im markhaltigen Theil der Retina, ist bedeckt non einem kleinen Blutgerinnsel im Glaskörper. - Nach 12 tägiger Erhärtung in Müler'scher Lösung fanden sich im ganzen Unfang des Wundrandes zahlreiche Varicosităten verschiedener Grösse und Form, bei den perlschnurartigen Bildungen ist jedesmal die startste Anschwellung dem Wundrande zugekehrt. Fig. 12 gibt eipe Atuzahl dieser Verdickungen, welche durch Zerzupfen frei präparirt waren. Einige sind noch in Verbindung mit Theilea der Markscheide. Bei $b$ ein runder Körper qbone Ausläufer, wahrscheinlich bel der Präpanation verstümmelt. a Eine Faser ebenfals mit Resten der Markscheide, die etwas entfernt vom Wundrande zwischen nonalen markhaltigen Nervenfasern gefonden wurde.

Exp. IX. 9. Mai 1871. Grosses Kaninchen.

3) Stich in den Jnken Bulbus: - Tod nach 48 Stouden. - Herzu Fig. 11.

Stichverletzung im marklosen Theil der Retina $2 \mathrm{Mtn}$. von der Papille. Ein kleines Coagulum ragt van bier in den Glaskörper. Im ganzen Umfang der Wunde, jedoch zahlrelcher am centralen als am peripherischen hande, gestielte Varicositäten. Bei $b$ wederum ein rundlicher Körper ohne Ausläluer (Zerzupfungspräparat nach 24stündiget Enwirkung ron Müller'scher Fläsiglaet und nachträglicher Im- 
bibition mit Carmin). Im weiteren Umfang um die Verletzung wurden keine Varicositäten beobachtet.

Exp. XIII, 29. October 1871. Mittelgrosses Kanincben.

4) Stich in den linken Bulbus. - Tod nach 77 Stunden.

$4 \mathrm{Mm}$. von der Papille ein kleines Loch im marklosen Theil der Retina. Untersuchung nach 4tägiger Behardlong mit der von Landois angewandten Mischung. Rings um die Stichöffnung eine mässige Zahl von spindelförmigen Anschwellungen in der Continuität der Faser und terminale mehr kuglige Verdickungetı.

Exp. XII. 11. Juni 1871. $\frac{a}{4}$ Jahr altes Kaninchen.

5) Einstich im linken Auge. - Tod nach 8 Tagen.

Stichöffnung $2 \mathrm{Mm}$. von der Papille im marklosen Theil der Retina. - Negativ.

Exp. VII. 26. April 1871. 3jähriger mittelgrosser Hund.

6) Stich in dea linken Bulbus. - Tod nach 10 Tagen.

Retina vollkommen transparent mit Ausnahme eines weissen Ringes um die Papille. Glaskörper und Linse getrübt. Stichöffnung im äusseren Umfang $3 \mathrm{Mm}$. binter der 0ra serrata. Nach 3tägiger Einwirkung von Müller'scher Flüssigkeit ond Zerzupfung fand sich blos ein grosser zwerchsackförmiger Körper ohne Kern, ganz von der Beschaffenheit der im anderen Auge reichlicher vorhandenen Elemente. - Ganglienzellen wohlerhalten.

7) Stich in den rechten Bulbus; nachträgliche Injection von Oleum crotonis misslang wegen Unruhe des Thieres. - Tod nach 10 Tagen. - Hierzu Fig. 10.

Glaskörper und Linse getrübt. Retina mit weissem Ring um die Papille. Stichöfinung $8 \mathrm{Mm}$. von der Papille in äusseren Umfang der Retina. Nach 24stündiger Einwirkung von Müller'scher Flüssigkeit wird die Partie um die Stichöffnung ausgeschnitten und mit der Innenfläche nach oben bei schwacher Vergrösserung durchsucht. Es fanden sich am Wundrande und in der nächsten Umgebung zahlreiche gruppenweise stehende kernlose kuglige Elemente. Durch Zerzupfen kommen einige blasse feingranulirte Ganglienzellen mit grossem Kern und zahlreiche mattglänzende fein- und grobgranulirte Varicositäten zum Vorschein, in denen auch nach Zusatz von Essigsäure, ein Kern nicht sichthar ist. Neben den ausgesprochenen grossen Formen (Fig. 10) kommen alle Stufen bis zu gewöbnlichen cadaverösen Varicosităten vor. - Elemente der Stäbchen - und Körnerschicht, Capillargefässe normal. Mäller'sche Fasern sind sehr entwickelt, einige zeigen mehrere Kerne.

Exp. XIV, 22. November 1871. Grosses Kaninchen.

8) Links Einstich mit glühender Nadel. Tod nach 16 Tagen.

$3 \mathrm{Mm}$. von der Papille entfernt eine $2 \mathrm{Mm}$. Durchmesser haltende 0efinung im marklosen Theil der Retina. Das Präparat, nach 2tägiger Einwirkung von Müller'scher Flüssigkeit zerzupft, ist viel derber als normal; enthält zahlreiche zum Theil verfettete Bindegewebszellen. Nervenfasern sparsam, mit Anschwellungen, die taicht über cadaveröse Varicosităten hinausgehen.

9) Rechts Stichverletzung. - Tod nach 16 Tagen. 
Kleines Loch in der Peripherie eines Markbüschels, $3 \mathrm{Mm}$. von der Papille. Nach 2tägiger Maceration in Jodserum ist die betreffende Stelle wie bei 8 ) derb, schwer zerzapfbar. Derselbe Befund wie im linken Auge; nur eine grössere feingranulirte kuglige Anschwellung in der Continuităt einer zarten Nervenfaser. Erbaltene markhaltige Fasem wurden nicht gefunden, blos Myelintropfen.

Aus diesen Versuchen geht hervor, dass varicöse Hyperw trophie der Nervenfasern der Retina durch traumatische Einwirkung beim Raninchen und Hund sich herrorrufen lässt; sie findet sich 1-16 Tage nach der Verletzung, beschränkt sich meist auf den Wundrand, and geht in jedem Fall bur ganz wenig uber denselben hinaus. Die Varicositäten entsprechen den von Berlin beim Menschen in Folge von Tranma beschriebenen Formen; die höchste Stufe der Entwickelang (ganglioforme Hypertrophie) wurde aicht beobachtet.

Auch im Gehirn lassen sich, wie aus einem Experiment des Herrn Prof. Grohe hervorgeht, die varicösen Nervenfasern künstlich erzeugen. Bei einem Kaninchen, dem Herr Prof. Grobe Ende November $1871 \mathrm{zu}$ anderen Zwecken eine Stichwunde in die rechte Hewispbäre beigebracht halle, und das 6 Tage nach der Operation starb, fand sich die nachste Ungebung des Wundkanals missfarbig und erweicht und milkoskopisch in dieser Partie ausser vermehrten Zellen der Neurogliá; Körnchenzellen, verfetteten Capillargefässen ausgesprochene Anschwellung der Axencylinder. Dieselben stehen im frischen mil Jodserum untersuchten Präparat noch in Zusammenhang mit markbaltigen Fasern, selbst einige innerhalb der Markscheide liagende Anschweliungen sind vorhanden. Nach mehrtägiger Einwirkumg won MUller' 'seber Flüssigkeit ist das Mark abgebröckelt, und die Varlcosităten finden sich meist als rupdliche and spindelförmige Kurper yor, wie sie Virchow (34) ans den Erweichungsbeerden bei Encephalitis neonatorum abgebildet hat - Auch in der erweichten grauen Substanz gelang es einzelne solcher spindelformigen Köper, freilich nieht im Zusammenhang mit Nervenfasern oder Ganglienzellea zu finden.

Ueber die monp holog g j che Bed e u tung der varicösen Hypertrophie sind von jeher die Ansichten getheilt gevesen. Die meisten Autoren sehen die Veränderung: als passive Ersobeinung an, als Degeneration der Nerwenfasern, bedingt entweder durch gestörte 
Blutmischung oder gestörte Circulation. Frommann (37, S. 112) betrachtet die Verdickung als eine secundäre Degeneration im Sinne yon Türck: die durch den interstitiellen Prozess functionsunfähig gemachte Nervenfaser erleide in der Richtung der Leitung eine Ernährungsstörung und schwelle dadurch an. Berlin (24, S. 308) sieht in der Zerreissung der Faser ein Hauptmoment: vielleicht seien die reichlichen Blntungen bei Retinitis ex Morbo Br. die Ursache der Ruptur von Nervenfasern und dadurch auch der Varicositätenbildung. So viel Einladendes diese Erklärungsversuche für einzelne Fälle haben, so reichen sie doch für andere keineswegs aus, wie man sich bei einer unbefangenen Prüfung der Casuistik sagen muss.

Die andere Anschanung, der ich mich anschliesse, fasst die Varicositätenbildung als einen activen Vorgang, als eine Hypertrophie der Nervenfasern auf. Virehow neigte schon in seiner ersten Publication $(2,3,4)$ zu dieser Auffassung und hob die Combination mit irritativen und entzündichen Local- und Allgemeinprozessen neulich (34) noch schärfer bervor. H. Mäller $(7,8,5,54)$ machte auf die mögliche Substitution der Varicositäten durch markhaltige Fasern in der Retina aufruerksain, und vermuthete, dass es sich in beiden Fällen um einen hypertrophischen Vorgang handle. Später (31) glaubte er unter analogen Verinaltnissen (bei Retinitis ex Morbo Br.) geradezu eine Wueherung von Ganglienmasse in der Choroides annehmen zu dürfen.

Leider ist H. Müller in dieser Angelegenheit über das Gebiet des Zweifels nicht hinausgekommen, und die neueren Angaben von Sebweigger (Graefe A. f. O. VI, 2. S. 309, 323) und Iwan off (Stricler's Handbuch der Gewebelehre S. 1042) lassen in Müller's Beobachtungen normale, wenn auch individuell variirende Bildungen vermuthen.

In letzter Zeit bin ich bei Gelegenheit einer anderen Untersuchung auf ein Object gestossen, welches, wie ich glaube, für die active Entstehung der Varicositäten verwerthet werden kann. In einem Spinalganglion der P. sacralis eines $28 \mathrm{Cm}$. langen Rindsfötus fanden sich neben wohlcharakterisirten (kernlosen) Axencylindern und (kernhaltigen) Ganglienzellen eigenthümliche längliche Körper, theils eingeschoben in den Verlauf eines Axencylinders, theils isolirt in der Flüssigkeit. Ferner mebriach gelappte und perlschnurartige Bildungen, die ganz ähnlich wie grössere Varicositäten in retinitischen 
Heerden sich ausnahmen. Der Hauptunterschied yon hypertrophischen Axencylindern war, dass in einigen djeser Anschwellungen (nicht in allen) ein Kern mit Kernkörperchen vorhanden war.

Es sind hier noch weitere Beobachtungen nothwendig, doch glaube ich jetzt schon annehmen zu düren, dass in diesen Bildungen des wachsenden Körpers das physiologische Paradigma für die pathologische Hypertrophie gegeben ist. Wenigstens für die jüngeren weichen Formen: die derben Bildungen mit kernăhnlichen $\mathbf{A b -}$ scheidungen sind wohl sicher ein degenerativer Zustand, welchem die jüngeren Formen nach kürzerem oder längerem Bestande verfallen können.

\section{Literatir.}

Retina: Fall 1. Heymann und Zenker, r. Graefe's Archiv $f_{n}$ Ophthalmologie II. 2, S.141. - F. 2, 3, 4. Virchow in diesem Archiv X. S. 171. 5. Lichtenstein ( orta Diss. Regiomont. 1857. - 6. Beckmann, Dies. Archiv XIII, S. 97. 7. 8. H. Muller in von Graefe's Archiv IV. 2. S. 42. - 9. H. Müller, Waraburg. medicin. Zeitschr. 1. S. 45. - 10. von Graefe und Schweigger in v. Graefe's Archiv VI. 2. 5.27\%. - 11. 12. 13, 14. Schweigger, ebenda S. 295. 15. Schweigger l. c, VIl. 2. S.63. - 16. 17. Wed1, Atlas der patholog. Histologie des Auges, Retina - Opticus. Taf. I. Fig. 2. Taf. II. Fig. 13. - 18. von Recklinghausen in Liebreich's Atlas der 0phthalmosc. 1863. Taf. X. Fig. 3. 19. Schelske in Schweigger, Vorlesungen über dea Augenspiegel 1864. S.109. 20. Virchow und von Graefe in Graefe's A. f. 0. XII. 2. \$. 117. (Fgl. Zehender's Monatsbi. 1864. S. 367). - 21. Koster in Zesde jaarlijksch Verslag etc. door Donders 1865. S. 8. - 22. Knapp in Graefe's Arch. XII. 1. S. 127. 23. Berlin, ebenda XIII, 2. S. 292. - 24. Berlin 1, c. XIV. 2. S.279. 25. 26. Leber, ebenda S. 341. 356. - 27. 28. Sehmidt und Wegner, ebenda Bd. XV. S. S. 262, 206. - 29. Roth, Dies Archip XLIX. S. 44h. 30. Roth, Berliner klinische Wochenschr. 1870. No. 43. 5. 520.

Zweifehaft ob hỉehergehörig: Wagner, Dies. Arch. Kull, \$.218. - Nagel, Graefe's Arch. ML 1. S. 191. - Manz, ebendas. XII. 1. S. 1.

Choroides: 31. H. Mäller, Würbg. Verhandlg. X. S. 189, vgl. S. 108. Würzg. medic. Ztschr. 1. \$. 53.

N. abducens: 32. Leber in Graefe's A. f. 0. XIV. 2. 3. 346.

Gebira, graue Substanz: 33. Virchow dies. Archiv X. \$.178. - weisse Substanz: 34. Virchow 1, c. XUV. S. 475. - Cerebellum, graue substanz: 35. Hadich 1, ELVI. S. 218.

Rüclseninark, weisse Substanz: 36 . J. Luys in Archives génér, de médecine. Nonbre. 1864. p. 513, - 37. Fromman $\mathrm{n}$, Untersuchungen über die nor- 
male und pathol. Anat. des Rückenmarks, 1864. S. $81 \mathrm{fgg} .(\mathrm{S} .98,105)$. 38. 39. Leyden im Archiv für Psychiatrie. Berlin 1870. II. S. 655, 664. 40. W. Muller, Beitr. z. pathol. Anat. und Physiol. des Rückenmarks. Leipzig 1871. S. $12 \mathrm{fg}$.

\title{
Erklärung der Abbildungen.
}

Tafel XII.

Vergrösserung 350.

Fig. 6-9 gebören zu Fall 1: verdickte Nervenfasern aus der grauen Substanz der Lendenanschwellung vom Menschen.

Fig. 10. Varicöse Nervenfasern aus der Retina vom Hund, 10 Tage nach der Verletzung. Exp. ViI.

Fig. 11. Eben solche vom Kaninchen, 48 Stunden nach der Verletzung. Exp. IX.

Fig. 12. Eben solche aus dem markhaltigen Theil der Retina vom Kaninchen, 28 Stunden nach der Verletzung. Exp. XV.

\section{VIII.}

\section{Arthritis deformans der beiden Schulter- und Ellbogengelenke und des linken Hïftgelenkes.}

\author{
Beschrieben von Dr. A. Weichselbaum, \\ Assistenten für pathologische Anatomie am Josephinum in Wien.
}

(Hierzu Taf. XIII. Fig. 1-2.)

Unsere Kenntnisse von der Artbritis deformans, ohnebin erst seit den dreissiger Jahren dieses Jahrbunderts datirend, sind ungeachtet vieler ausgezeichneter Schilderungen dieser Krankheit noch in mancher Beziehung lückenhaft, weshalb ich die Veröffentlichung besonders prägnanter Fälle und somit auch des vorliegenden Falles für nicht ganz unnütz erachte. -

Er betraf einen 53jährigen verheiratheten Mann, welcher Geschäftsfübrer in einem Meerschaumgeschäfte und zugleich Mitglied eines hiesigen Vorstadt-TheaterOrchesters war und vor seiner Erkrankung in sebr guten Verbältnissen lebte. Die Krankheit soll nach der Aussage des Kranken vor 10 Jahren hegonnen haben, als er nehmlich einmal rom Theater nach Hause gehend plötzlich zusammenstürzte 